\title{
Adherencia a la medicación antipsicótica en pacientes indígenas con esquizofrenia
}

\author{
Alejandra Caqueo-Urízar, ${ }^{1}$ Alfonso Urzúa $M,{ }^{2}$ Claudia Miranda-Castillo, ${ }^{3}$ Matías Irarrázaval|4,5
}

Artículo original

\section{ABSTRACT}

\section{Introduction}

Non-adherence to antipsychotic medication remains a complex problem in the treatment of schizophrenia patients, especially in indigenous population.

\section{Objective}

The aim of the study was to assess the differences in drug adherence, measured by the attitude towards the antipsychotics among Aymara and Non-Aymara patients with schizophrenia.

\section{Method}

The sample consisted of patients receiving treatment in the Mental Health Public Services in Bolivia (32.8\%), Peru (33.6\%) and Chile (33.6\%). We used the Drug Attitude Inventory (DAI-10); the Barnes Akathisia Scale (BAS), as a measure of side effects, and the Positive and Negative Syndrome Scale (PANSS) to assess the severity of the disorder.

\section{Results}

The findings showed that Aymara patients present less adherence than Non-Aymara people; however, these differences were not significant $(t=1.29 ; p=0.19)$. The severity of the disorder, as well as the age, showed a significant association with adherence, revealing that younger patients and with greater symptoms presented a more negative attitude toward the drugs.

\section{Discussion and conclusion}

The lack of significant differences between the groups responds to three possible reasons: 1. This sample of indigenous patients is integrated on Mental Health Services that offer a clear biomedical approach where drug therapy is the primary treatment. 2 . It is possible that these indigenous patients are changing their conception of mental disorder, and 3. A significant number of families have migrated to urban areas. These migratory dynamics have promoted the loss of traditions and customs of the ethnic group, which gradually adopts new and intercultural lifestyles. Professionals should be warned about applying stereotypes regarding the relationship between ethnicity and antipsychotics.

\section{RESUMEN}

\section{Introducción}

La falta de adherencia a la medicación antipsicótica sigue siendo un problema en el tratamiento de pacientes con esquizofrenia, más aún en población indígena.

\section{Objetivo}

Evaluar las diferencias en la adherencia farmacológica, medida a través de la actitud hacia los antipsicóticos, entre pacientes aymara y no-aymara con esquizofrenia.

\section{Método}

La muestra estuvo compuesta por pacientes que recibían tratamiento en los Servicios Públicos de Salud Mental de Bolivia (32.8\%), Perú (33.6\%) y Chile (33.6\%). Se utilizó el Inventario de Actitud hacia la Medicación (DAl-10); la Escala Barnes de Acatisia (EBA) como medida de efectos secundarios y la Escala para el Síndrome Positivo y Negativo de la Esquizofrenia (PANSS) para evaluar la severidad del trastorno.

\section{Resultados}

Los pacientes aymara presentan una menor adherencia que los pacientes no-aymara, sin embargo, estas diferencias no fueron significativas $(t=1.29 ; p=0.19)$. La severidad del trastorno y la edad mostraron una asociación significativa con la adherencia, observándose que pacientes más jóvenes y con mayor sintomatología presentan una actitud más negativa hacia los fármacos.

\section{Discusión y conclusión}

Se discute cómo las dinámicas migratorias han promovido la pérdida de tradiciones y costumbres propias de la etnia promoviendo la adopción de estilos de vida nuevos y cada vez más interculturales, e incluso cambiando su concepción de la enfermedad mental.

Los profesionales tratantes deben estar conscientes de no aplicar estereotipos en cuanto a la relación etnia-antipsicóticos.

Palabras claves: Indígenas, esquizofrenia, adherencia, medicación

Key words: Indigenous, schizophrenia, adherence, medication.

Escuela de Psicología y Filosofía, Universidad de Tarapacá, Arica, Chile.

2 Escuela de Psicología, Universidad Católica del Norte, Chile.

Instituto de Sociología, Pontificia Universidad Católica de Chile.

Departamento de Psiquiatría, Facultad de Medicina, Hospital Clínico, Universidad de Chile.

Instituto Milenio para la Investigación en Depresión y Personalidad, Santiago, Chile.

Correspondencia: Dra. Alejandra Caqueo-Urízar. Escuela de Psicología y Filosofía, Universidad de Tarapacá. Avenida 18 de Septiembre 2222, Arica, Chile. Tel: (56-58) 220 - 5622. E-mail: acaqueo@uta.cl

Recibido primera versión: 18 de diciembre de 2015. Segunda versión: 7 de septiembre de 2016. Aceptado: 26 de septiembre de 2016. 


\section{INTRODUCCIÓN}

La medicación antipsicótica ha demostrado reducir la sintomatología y las recaídas asociadas a la esquizofrenia. ${ }^{1}$ A pesar de estos beneficios, las tasas de no-adherencia por parte de los pacientes oscilan entre un $20 \%$ y $89 \%{ }^{2-10}$ siendo éste, el mayor obstáculo en el manejo del trastorno. ${ }^{2}$

La adherencia se define como "el grado en el que la conducta de un paciente, en relación con la toma de medicación, el seguimiento de una dieta o la modificación de hábitos de vida, se corresponde con las recomendaciones acordadas con el profesional sanitario". ${ }^{11}$ Por otro lado, el término cumplimiento terapéutico se relaciona con una conducta más pasiva y sumisa por parte del paciente a obedecer una orden o prescripción médica. La carencia de participación en la definición, según Dilla et al. ${ }^{12}$ (2009) estaría evidenciando el menor uso del término cumplimiento aunque en la clínica ambos conceptos se usan indistintamente..$^{13}$

La adherencia al tratamiento farmacológico en la esquizofrenia en un fenómeno complejo y de múltiples factores, resumidos en cuatro: variables sociodemográficas; variables relacionadas con el trastorno propiamente tal (tipo de esquizofrenia, severidad, curso del trastorno); variables relacionadas con el tratamiento (frecuencia e intensidad de los efectos secundarios, duración del tratamiento) y finalmente, variables que tienen que ver con los valores y actitudes del paciente (actitud hacia la enfermedad). ${ }^{8,14,15}$ Los pacientes que no mantienen una adecuada adherencia farmacológica presentan alrededor de 10 veces mayor riesgo de recaídas y cuatro veces mayor riesgo de hospitalización que aquellos que tienen una adecuada adherencia. ${ }^{16}$ Esto último tiene consecuencias tanto para el paciente como para la sociedad. ${ }^{17-20}$ Estudios previos han encontrado que pacientes varones, jóvenes, con bajo nivel socioeconómico, pertenecientes a una minoría, con pobre funcionamiento social y con dificultades en el establecimiento de una adecuada alianza terapéutica presentan mayores dificultades para lograr adherencia en el tratamiento. ${ }^{8,21}$ Se ha observado de igual forma que el contexto cultural y la etnicidad también tienen una influencia importante. Así, pacientes afro-americanos y mexicanos-americanos mostraron más baja adherencia (19 días y 18 días menos, respectivamente) que pacientes blancos en un estudio realizado en Texas. ${ }^{22,23}$ En esta misma línea, Diaz et al., ${ }^{24}$ en el 2005, encontraron que pacientes afro-americanos y latinos monolingües también presentaban peor adherencia que los caucásicos. Similares resultados fueron encontrados en San Diego. ${ }^{25}$ Esto último lleva a plantear que la adherencia a la medicación antipsicótica varía entre los grupos étnicos. ${ }^{1}$

Si bien los estudios recién mencionados se han llevado a cabo en Estados Unidos, existe escasa bibliografía en Latino-América que permita establecer una relación entre la adherencia a los fármacos antipsicóticos y la pertenencia a las minorías étnicas. Sin embargo existen en la región algu- nas experiencias con intervenciones psicosociales que mejoran la adherencia de pacientes con esquizofrenia, como es la incorporación de entrenamiento de habilidades sociales, la rehabilitación psicológica, el tratamiento integrado y la psicoeducación. ${ }^{26-30}$

El presente artículo es un estudio observacional transversal que tiene como objetivo describir el grado de adherencia al tratamiento farmacológico en pacientes con esquizofrenia pertenecientes a la etnia indígena aymara y compararlo con el grado de adherencia con pacientes no-aymara. Se estudiaron además el efecto de las variables sociodemográficas en la adherencia al tratamiento. Para el logro del objetivo se utilizó el Inventario de Actitud hacia la Medicación (DAI-10) ${ }^{31}$ así como la Escala Barnes de Acatisia (EBA) ${ }^{32}$ como medida de efectos secundarios y la Escala para el Síndrome Positivo y Negativo de la Esquizofrenia ${ }^{33}$ para evaluar la severidad del trastorno.

Ambos grupos de pacientes, tanto aymara como no-aymara recibieron tratamiento en los Servicios de Salud Mental en Bolivia, Perú y Chile. La comunidad aymara presenta una población aproximada de dos millones de personas radicadas en el Altiplano de la Cordillera de los Andes. Sin embargo, recientes generaciones de aymara han migrado masivamente desde los pueblos rurales hacia ciudades más grandes y con mejores perspectivas de trabajo. ${ }^{34-37}$

La cosmovisión aymara ordena el mundo según tres dimensiones: relaciones sociales, relaciones con las "divinidades" y la relación con la naturaleza. Estas tres dimensiones están íntimamente entrelazadas y se encuentran plenamente integradas en los paisajes y en la naturaleza en general. Se puede decir que su modo de comprensión del universo se articula en torno a procesos "cíclicos" de la propia naturaleza y del calendario ritual que ellos mismos han ido adecuando a los ritmos de la naturaleza.

Los aymara no se ven a sí mismos como dueños de la naturaleza, sino más bien como parte intrínseca de ella, fundamental para la mantención (ritual, educacional, económica) de la armonía deseada. El principio narrativo de los aymara que concibe "el buen vivir" como un caminar en armonía, es posible por un concepto de cultura entendida históricamente de manera dinámica y cohesionada. ${ }^{35}$

Si se considera esta concepción dinámica de la tradición aymara se puede afirmar que su actuar va a estar relacionado con la necesidad de preservar y mantener el equilibrio y la armonía. Si se puede hablar de una ética aymara, ésta supondría fundamentalmente una práctica y una vivencia comunitaria, distinta a la ética que se ha vuelto hegemónica en Occidente, que se basa en el individualismo y el logro personal. Es así como los ambientes urbanos a menudo llegan a romper con la concepción de equilibrio y armonía propios de la cosmovisión andina. ${ }^{38}$

En la hipótesis del estudio se espera encontrar diferencia en relación a la adherencia hacia la medicación entre los grupos y el efecto de las variables sociodemográficas. De esta for- 
ma los pacientes aymara, quienes consideran que la enfermedad es generada por una falta de equilibrio provocado por los malos espíritus o un pecado de la persona afectada, mostrarán una menor adherencia hacia la medicación antipsicótica, esto también dado porque el tratamiento en esta comunidad implica la ingesta de hierbas y la realización de rituales. ${ }^{39,40}$

\section{MÉTODO \\ Participantes}

La muestra estuvo compuesta por pacientes con esquizofrenia que recibían tratamiento en tres centros de salud mental: Tacna (Perú), La Paz (Bolivia) y Arica (Chile).

Se incluyó a pacientes aymara y no-aymara. Ambos grupos vivían en las mismas zonas urbanas y eran atendidos por los mismos centros de salud mental, presentando además características socio-demográficas similares, tales como bajo nivel educativo y ocupacional. En relación a cada centro de salud mental que participó en el estudio, estos presentaban características similares en cuanto a tamaño, tipo de tratamiento que se otorgaba a los pacientes, tipo de profesionales tratantes y gratuidad de la atención. Los pacientes aymara hablaban castellano y su lengua nativa. En el reclutamiento de los pacientes, la primera autora revisó las listas de pacientes que asistían a cada centro en cada ciudad y el equipo de investigación hizo evaluaciones durante un período de tres meses en cada país. Los pacientes aymara fueron identificados por sus apellidos según lo establecido por la legislación sobre los pueblos indígenas en los tres países, o por autodefinición. Los sujetos fueron invitados a participar cuando asistían a sus visitas de control mensual ya que recibían tratamiento ambulatorio. Se excluyeron aquellos pacientes que se encontraban en crisis psicótica o aquellos que presentaban algún trastorno sensorial o cognitivo. La muestra final incluyó a 253 pacientes que aceptaron participar, diagnosticados con esquizofrenia de acuerdo al CIE- $10^{41}$ por su psiquiatra tratante $(33.6 \%$ de Chile, $33.6 \%$ de Perú y $32.8 \%$ de Bolivia). Las entrevistas se realizaron entre mayo de 2012 y febrero de 2013.

\section{Procedimientos}

El estudio fue aprobado por el Comité de Ética de la Universidad de Tarapacá y por el Servicio Nacional de Salud de Chile. Dos psicólogos externos, supervisados por la investigadora principal, llevaron a cabo las evaluaciones de los pacientes con una duración de entre 30 y 40 minutos. Éstas implicaban una entrevista para recabar información demográfica y clínica, así como la aplicación de los cuestionarios detallados más adelante. Antes del inicio de la entrevista se solicitó la firma del consentimiento informado. Se les explicaron los objetivos del estudio, así como el carácter volunta- rio de la participación. No se ofreció ninguna compensación por participar en el estudio.

El estudio cumple con los principios éticos contenidos en la Declaración de Helsinki (2008) de la Asociación Médica Mundial.

\section{Instrumentos}

Inventario de Actitud hacia la Medicación (DAI-10): ${ }^{31}$ Escala de auto-reporte de 10 ítems que fue desarrollada para evaluar las actitudes, experiencias y creencias sobre los fármacos antipsicóticos. El DAI-10 se considera un buen predictor de la adherencia al tratamiento en pacientes con esquizofrenia. ${ }^{31,42}$ Las puntuaciones varían de -10 (actitud muy mala) a 10 (mejor actitud posible). Una puntuación total positiva indica una actitud positiva hacia la medicación y una puntuación total negativa indica una actitud negativa. Pacientes con puntaje de seis a 10 se consideran como adherente, de cero a cin$\mathrm{co}$, moderado y en los rangos negativos se considera como no-adherente..$^{43}$ Este instrumento cuenta con una versión en castellano llevada a cabo por Ramírez et al. ${ }^{44}$ en el año 2004. En relación a sus propiedades psicométricas este instrumento muestra un índice de confiabilidad interevaluadores de 0.61 $(p<0.001)$ y un coeficiente de consistencia interna de 0.57 . La versión en español del DAI muestra validez convergente así como una moderada confiabilidad. ${ }^{44}$ Las puntuaciones que se analizan en este estudio fueron obtenidas de los pacientes.

Escala Barnes de Acatisia (EBA): ${ }^{32}$ Escala de cuatro ítems que evalúa la presencia y gravedad de la acatisia inducida por fármacos. Debe ser administrada por el clínico e incluye ítems objetivos y subjetivos, junto a una evaluación clínica global de acatisia. Las puntuaciones, para cada uno de los tres primeros ítems, oscilan de 0 (ausente) a 3 (acatisia grave). El cuarto ítem es para calificar la gravedad global. Para cada puntuación se proporcionan definiciones comprensivas. Evalúa el estrés causado al paciente por un síntoma específico, el que se puntúa utilizando la evaluación clínica global. Las puntuaciones que se analizan en este estudio fueron obtenidas a partir de la valoración del psiquiatra o personal médico a cargo del paciente. Este instrumento presenta un coeficiente de fiabilidad de 0.74 para el ítem objetivo, 0.73 para el de intranquilidad subjetiva, de 0.90 para el disconfort subjetivo y 0.96 para la valoración global.

Escala para el Síndrome Positivo y Negativo de la Esquizofrenia (Positive and Negative Syndrome Scale, PANSS): ${ }^{33}$ Consta de 30 ítems que evalúan el síndrome esquizofrénico. Cada ítem se puntúa según una escala Likert de siete grados de intensidad o gravedad. En esta escala el 1 siempre equivale a ausencia del síntoma y el siete a presencia con una gravedad extrema. Este instrumento consta de tres subescalas de diferentes síntomas: positivo, negativo y psicopatología general. La Escala PANSS ha sido traducida y validada en España por Peralta y Cuesta $(1994)^{45}$ y también Fresán et al. $(2005)^{46}$ examinaron las propiedades psicométricas de este 
instrumento en México. Este instrumento presenta buena validez inter-observador y de constructo, elevada consistencia interna (alfa de Cronbach de 0.73 para la escala positiva, de 0.83 para la negativa y de 0.87 para la de psicopatología general) y adecuada estabilidad test-retest. El coeficiente de correlación intraclase es de alrededor de 0.80 para las tres subescalas.

Características demográficas y clinicas: Las características demográficas del paciente incluyeron sexo, edad, origen étnico (aymara y no-aymara), nivel educativo (bajo o alto), estado civil (con pareja o sin pareja), ocupación e ingresos familiares (medida del salario total mensual de todos los miembros de la familia, expresados en dólares). Las características clínicas incluyeron severidad del trastorno, medida por la Escala PANSS, el número de hospitalizaciones en los últimos tres años y la medición de los efectos secundarios de la medicación por medio de la Escala de Acatisia de Barnes (rango de la escala: de 0 a 5). El umbral determinado para el diagnóstico de acatisia fue de una puntuación total de dos o más.

\section{Análisis estadístico}

Primero se utilizó una prueba de $t$ para evaluar diferencias en la puntuación del DAI-10 entre pacientes aymara y no-aymara. Posteriormente, para investigar otras variables sociodemográficas que podrían explicar las diferencias en adherencia a la medicación entre los dos grupos, se utilizó un modelo de regresión lineal controlando las características sociodemográficas y clínicas de los pacientes. Cuando fue posible se utilizaron las variables de resultado como escalas contínuas. Los datos se analizaron con SPSS versión 17.0 .

\section{RESULTADOS}

Doscientos cincuenta y tres pacientes con esquizofrenia participaron en el presente estudio. La edad media fue de 35.6 años, $66.4 \%$ eran hombres y $46.2 \%$ de los pacientes eran ay-

Tabla 1

Características sociodemográficas y clínicas de pacientes Aymara, No-Aymara y muestra total

\begin{tabular}{|c|c|c|c|}
\hline \multirow[b]{2}{*}{ Variables sociodemográficas } & \multicolumn{3}{|c|}{ Categoría (\%) } \\
\hline & Muestra total & Aymara & No-Aymara \\
\hline \multicolumn{4}{|l|}{ Sexo } \\
\hline Hombre & 66.0 & 66.0 & 67.0 \\
\hline Mujer & 34.0 & 34.0 & 33.0 \\
\hline Etnicidad & & 46.2 & 53.8 \\
\hline \multicolumn{4}{|l|}{ Estado civil } \\
\hline Casado & 6.3 & 6.8 & 5.9 \\
\hline No casado & 93.7 & 93.2 & 94.1 \\
\hline \multicolumn{4}{|l|}{ Ocupación fuera del hogar } \\
\hline Con ocupación & 30.4 & 31.0 & 31.3 \\
\hline Sin ocupación & 69.6 & 69.0 & 68.7 \\
\hline \multicolumn{4}{|l|}{ Nivel educativo } \\
\hline Bajo (< 12 años) & 84.2 & 86.3 & 82.4 \\
\hline \multirow[t]{3}{*}{ Alto ( $\geq 12$ años) } & 15.8 & 13.7 & 17.6 \\
\hline & Media (DT) & & \\
\hline & Muestra total & Aymara & Non-Aymara \\
\hline Edad & $35.6(12.4)$ & $33.9(11.5)$ & $36.9(13.1)$ \\
\hline $\begin{array}{l}\text { Ingreso familiar mensual } \\
\text { (US dólares) }\end{array}$ & $417.6(429.4)$ & $295.1(218.9)$ & $536.7(538.3)$ \\
\hline Severidad - Total PANSS* & $71.3(28.2)$ & $73.0(29.2)$ & $69.9(27.3)$ \\
\hline $\begin{array}{l}N^{0} \text { de hospitalizaciones } \\
\text { en los últimos } 3 \text { años }\end{array}$ & $1.2(1.5)$ & $1.3(1.4)$ & $1.1(1.5)$ \\
\hline EAB Total & $0.8(1.9)$ & $1.0(2.1)$ & $0.7(1.8)$ \\
\hline Criterios objetivos & $0.2(0.5)$ & $0.2(0.5)$ & $0.1(0.5)$ \\
\hline Conciencia de inquietud & $0.2(0.4)$ & $0.2(0.5)$ & $0.1(0.3)$ \\
\hline Malestar por inquietud & $0.2(0.5)$ & $0.2(0.5)$ & $0.1(0.5)$ \\
\hline Valoración clínica global & $0.2(0.6)$ & $0.2(0.7)$ & $0.1(0.5)$ \\
\hline DAl-10 & $3.0(4.8)$ & $2.6(4.9)$ & $3.4(4.6)$ \\
\hline
\end{tabular}

Nota: DT = Desviación Standard; PANSS = Escala para el Síndrome Positivo y Negativo de la Esquizofrenia; *PANSS puntuación total (PANSS $58=$ Leve; PANSS $75=$ Moderado; PANSS $95=$ Moderado-Severo; PANSS 116 = Severo); DAI-10 = Inventario de Actitud hacia la Medicación. $E A B=$ Escala de Acatisia de Barnes. 
mara. La severidad de los síntomas fue moderada, con una puntuación total de PANSS de 71.3 (DT = 28.2). La media en la Escala de Barnes fue de $0.87(\mathrm{DT}=1.97)$ por debajo del umbral de diagnóstico de acatisia. Los pacientes aymara presentan una puntuación significativamente mayor en la subescala de Conciencia de inquietud $(t=-2.09 ; p=0.03)$.

Los puntajes obtenidos en el DAI-10 reflejan una adherencia moderada hacia la medicación en la muestra total y una falta de adherencia en el $19.8 \%$ de los sujetos. Los pacientes aymara presentan menor adherencia que los pacientes no-aymara, sin embargo, estas diferencias no fueron significativas $(t=1.29 ; p=0.19)$. Esto se refleja en el alto porcentaje de la muestra con uno o más ingresos hospitalarios en los últimos tres años (59\% total, 69\% para aymaras).

Algunas características adicionales de los pacientes aymara y no-aymara se presentan en la tabla 1.

El análisis de regresión se presenta en la tabla 2. Una vez controlados los factores socio-demográficos y clínicos de los pacientes, se observa que la etnia no presenta una relación significativa con la adherencia al tratamiento farmacológico. Se destaca una asociación significativa entre el DAI-10 y la severidad del trastorno medida por el PANSS, observándose que los pacientes más severos presentan una menor adherencia al tratamiento antipsicótico, $\beta=-1.56$ $(-2.34,-0.77)$. De igual forma, la variable edad mostró una

\section{Tabla 2}

Modelo de Regresión Lineal del Inventario de Actitud hacia la Medicación (DAl-10)

\begin{tabular}{lc}
\hline Coeficiente de regresión $(95 \% \mathrm{IC})$ & DAl-10 \\
\hline Etnia* & $-0.09(-1.47,1.29)$ \\
Aymara & \\
No-aymara (Ref) & $-1.45(-2.97,0.07)$ \\
Sexo* & \\
$\quad$ Mujer & $1.41(0.67,2.14)^{* *}$ \\
Hombre (Ref) & $1.67(-1.35,4.70)$ \\
Edad & \\
Estado civil* & \\
Sin pareja & $-0.83(-2.30,0.63)$ \\
Con pareja (Ref) & \\
Ocupación* & \\
No & $0.40(-1.53,2.35)$ \\
Sí (Ref) & \\
Nivel educativo* & \\
$\geq 12$ years & \\
$<12$ years (Ref) & $0.22(-0.45,0.91)$ \\
Ingreso familiar mensual\# (US Dólares) & $0.00(-0.71,0.72)$ \\
$N^{\circ}$ de hospitalizaciones & \\
en los últimos 3 años & $-1.56(-2.34,-0.77)^{* *}$ \\
PANSS Total\# & $0.00(-0.79,0.81)$ \\
EAB Total &
\end{tabular}

Nota: IC = Intervalo de Confianza; ${ }^{*}=$ Coeficiente beta no estandarizado; $\#$ = Coeficiente beta Estandarizado; Ref = Grupo de referencia. ${ }^{*}{ }^{*} p \leq 0.01$. PANSS = Escala para el Síndrome Positivo y Negativo de la Esquizofrenia; DAI-10 = Inventario de Actitud hacia la Medicación. EAB = Escala de Acatisia de Barnes. asociación significativa con el DAI-10, pacientes más jóvenes mostrarían una menor adherencia al tratamiento farmacológico $\beta=1.41(0.67,2.14)$.

\section{DISCUSIÓN Y CONCLUSIÓN}

Los resultados muestran que, en general, tanto los pacientes indígenas como los no-indígenas presentan una actitud positiva hacia la medicación lo que implica una adherencia moderada hacia los antipsicóticos.

Los hallazgos permiten confirmar parcialmente la hipótesis del estudio. Si bien los pacientes indígenas presentan menos adhesión que los pacientes no-indígenas, las diferencias no son significativas. Estos resultados se podrían relacionar con el hecho de que los pacientes indígenas de esta muestra se encuentran integrados a los Servicios de Salud Mental que ofrecen un abordaje biomédico. Si bien, existe la voluntad de desarrollar un tratamiento biopsicosocial, en la práctica, esto se limita, la mayoría de las veces, a una aproximación más bien médica, con un fuerte énfasis en el tratamiento farmacológico, hospitalización breve en caso de crisis psicótica y seguimiento ambulatorio. Las intervenciones psicosociales aún son escasas en estos contextos. ${ }^{47}$

Otro abordaje a los resultados obtenidos abre la posibilidad de que estos pacientes indígenas estén cambiando su concepción de enfermedad. Estudios previos en esta misma población han mostrado que, contrario a lo que podría esperarse, los pacientes aymara no presentaron mayores creencias mágico-religiosas en torno a la esquizofrenia, más bien, estos pacientes atribuyeron causalidad a factores psicosociales. Estos últimos factores se relacionan con dificultades en aspectos económicos, carencia de empleo, bajo nivel educativo, entre otros. Así, estos pacientes aymara estarían incorporando un modelo de causalidad diferente al presentado por generaciones previas. ${ }^{48}$

Una tercera razón que podría explicar los hallazgos de este estudio apuntan a la dinámica migratoria que se ha generado durante las últimas décadas. Un importante número de familias han migrado hacia zonas urbanas. Estas dinámicas interculturales han conducido a una crisis de identidad en este grupo de personas, quienes, por diversas razones ya no se identifican como pertenecientes a este grupo étnico. ${ }^{37}$ Un estudio afirma que la población aymara, en el contexto andino chileno ha pasado de habitantes monolingües aymara a hablantes monolingües castellanos. ${ }^{49}$ Estos cambios en la cultura y en la sociedad, ha promovido la pérdida de tradiciones y costumbres propias de la etnia, adoptando poco a poco estilos de vida nuevos y cada vez más interculturales. ${ }^{50}$

Desde nuestra mirada como investigadores y clínicos, los resultados de este estudio son positivos. En la medida en que se mantenga una adecuada adherencia farmacológica se reducen las recaídas, se obtiene un mejor curso sintomatológico e incluso promueve una mejor interacción social. ${ }^{51,52}$ 
Cabe destacar que en la misma línea de estudios anteriores, ${ }^{53-57}$ la severidad del trastorno presenta una asociación importante en la adherencia a la medicación. Si bien no se puede establecer causalidad, es posible que los pacientes más gravemente afectados presenten una menor adherencia, sin embargo, es posible también que al presentar menor adherencia se descontinúa el tratamiento y, por ende, el paciente recae (mayor severidad).

La variable edad, al igual que en investigaciones previas, ${ }^{8,58}$ se posiciona como un factor importante, así, pacientes más jóvenes mostrarían una menor adherencia, de esta forma estos pacientes se constituyen como un grupo de riesgo. ${ }^{59}$ Otras variables sociodemográficas no estuvieron relacionadas con la adherencia, al igual que lo obtenido por Lacro et al. en el 2002. ${ }^{5}$ Así como tampoco se encontró que los efectos secundarios a la medicación tuvieran un impacto en las puntuaciones del DAI-10. Este resultado es similar a los encontrados por Perkins et al., 2006; ${ }^{60} 2008^{61}$ y Vassileva \& Milanova en el 2012. ${ }^{62}$

El estudio presenta una serie de limitaciones. Primero, el diseño transversal utilizado en esta investigación no permite establecer relaciones de causalidad, por lo que se hace necesario realizar estudios longitudinales. Segundo, la ausencia de diferencias entre pacientes aymara y no-aymara tal vez se relacione con la selección de la muestra. De hecho, hemos comparado las actitudes hacia la medicación en pacientes que reciben tratamiento en los mismos Servicios de Salud Mental, sin embargo, no podemos extrapolar nuestros resultados a todos los pacientes aymara y especialmente para quienes presentan problemas de acceso a los servicios, muchos de estos pacientes aún residen en el Altiplano. Tercero, la medición de actitudes hacia la medicación se basó en una valoración subjetiva de la misma y no contó con mediciones más objetivas. Cuarto, el estudio no contó con una clasificación del tipo de antipsicótico utilizado por los pacientes, futuras investigaciones deberían considerar diferencialmente entre el efectos de los antipsicóticos clásicos y los atípicos, así como incorporar otros elementos relacionados con la adherencia, tales como la alianza terapéutica, el tiempo de evolución de la psicosis no tratada, el apoyo familiar y social. Así también se podría evaluar si el uso de antipsicóticos atípicos inyectables de acción prolongada pudiera ser mejor tolerado por pacientes pertenecientes a la etnia. Por otro lado, la información obtenida no permitió la consideración de los componentes psicosociales o las necesidades psicoeducativas de los sujetos, que son factores que están asociados a la adherencia terapéutica.

A pesar de las limitaciones mencionadas, los resultados muestran una menor adherencia farmacológica en los pacientes indígenas, y una actitud positiva global hacia la medicación, que si bien no es estadísticamente significativa podría ser un indicador de que en la población existen diferencias reales, lo que debe demostrarse en otro estudio. Los profesionales tratantes deben estar conscientes de no aplicar estereotipos en cuanto a la relación etnia-psicofármacos, siendo indispensable incorporar intervenciones psicoterapéuticas y psicoeducativas que aborden esta temática en los centros de salud mental de estos países. De igual forma, se espera que los profesionales tratantes incorporen elementos claves desde la psiquiatría transcultural, así también que estos elementos sean abordados desde su formación.

Tanto pacientes, familiares y profesionales deben otorgar la importancia que radica en el establecimiento de una adecuada adherencia al tratamiento para lograr, finalmente, una mejor calidad de vida.

\section{Financiamiento}

Esta investigación fue financiada por la Universidad de Tarapacá por medio del Proyecto Mayor de Investigación Científica y Tecnológica UTA n 3732-16 y por el Convenio de Desempeño UTA-MINEDUC.

\section{Conflicto de intereses}

Los autores declaran no tener ningún conflicto de intereses.

\section{Agradecimientos}

Agradecemos a Felipe Ponce y Jorge Escudero por su colaboración en este estudio y también las siguientes personas y servicios: doctores Hugo Sánchez, Ricardo Alvites, Andrés Collado, a Gladys Coaquira, Vilma Liendo y especialmente al doctor José Revilla del Hospital Hipólito Unanue de Tacna, Perú; a los doctores Marcio Soto, desde Arequipa, Perú; Fernando Garitano, Mauricio Peredo y Mabel Romero del Centro de Rehabilitación y Salud Mental San Juan de Dios; al Director del Hospital Psiquiátrico de la Caja Nacional de Salud de La Paz, Bolivia. También agradecemos a la doctora Magdalena Gardilcic, Ester López y Alejandra Lagos de Servicio de Salud de Arica, Chile.

Extendemos además un especial agradecimiento a todos los pacientes y sus familiares que participaron en este proyecto. Finalmente, un agradecimiento especial al doctor Stephen Gilman del National Institutes of Health (NIH) de Estados Unidos y al doctor David Williams de la Universidad de Harvard quienes colaboraron en el desarrollo de esta investigación.

\section{REFERENCIAS}

1. Gilmer TP, Ojeda VD, Barrio C, Fuentes D et al. Adherence to antipsychotics among Latinos and Asians with schizophrenia and limited english proficiency. Psych Serv 2009;60(2):175-182.

2. Nitzan U, Bukobza G, Aviram, S, Lev-Ran $S$ et al. Rebelliousness in patients suffering from schizophrenia-spectrum disorders - a possible predictor of adherence. Psychiatr Res 2013;209:297-301.

3. Fenton WS, Blyler CR, Heinssen RK. Determinants of medication compliance in schizophrenia: empirical and clinical findings. Schizophr Bull 1997;23:637-651.

4. Jeste SD, Patterson, TL, Palmer BW, Dolder CR et al. Cognitive predictors of medication adherence among middle-aged and older outpatients with schizophrenia. Schizophr Res 2003;63:49-58.

5. Lacro JP, Dunn LB, Dolder CR, Leckband SG et al. Prevalence of and risk factors for medication nonadherence in patients with schizophrenia: a comprehensive review of recent literature. J Clin Psychiatry 2002;63(10):892-909. 
6. Razali MS, Yahya H. Compliance with treatment in schizophrenia: a drug intervention program in a developing country. Acta Psychiatr Scand 1995;91:331-335.

7. Sun SX, Liu GG, Christensen DB, Fu AZ. Review and analysis of hospitalization costs associated with antipsychotic nonadherence in the treatment of schizophrenia in the United States. Curr Med Res Opin 2007; 23:2305-2312.

8. Velligan DI, Weiden PJ, Sajatovic M, Scott J et al. The expert consensus guideline series: adherence problems in patients with serious and persistent mental illness. J Clin Psychiatry 2009;70(4):S1-S46.

9. Young JL, Zonana HV, Shepler L. Medication noncompliance in schizophrenia: codification and update. Bull Am Acad Psychiatry Law 1986;14:105-122.

10. Lieberman JA, Stroup TS, McEvoy JP, Swartz MS et al. Clinical antipsychotic trials of intervention effectiveness (CATIE) investigators. Effectiveness of antipsychotic drugs in patients with chronic schizophrenia. N Engl J Med 2005;353(12):1209-1223.

11. World Health Organization. Adherence to long-term therapies evidence for action. World Health Organization. 2003;107-114. http://www. who.int/bookorders/anglais/detart1.isp?sesslan $=1 \& \quad$ codlan $=1 \&$ codcol=15\&codcch-526. Accessed October 19, 2010.

12. Dilla T, Valladares A, Lizán L, Sacristán JA. Adherencia y persistencia terapéutica: causas, consecuencias y estrategias de mejora. Aten Primaria 2009;41:342-348.

13. López San Román A. Treatment adhesion, treatment adherence or treatment compliance? Rev Clin Esp 2006;206:414.

14. Medina E, Salvà J, Ampudia R, Maurino J et al. Short-term clinical stability and lack of insight are associated with a negative attitude towards antipsychotic treatment at discharge in patients with schizophrenia and bipolar disorder. Patient Prefer Adherence 2012;6:623-629.

15. Fleischhacker WW, Oehl MA, Hummer M. Factors influencing compliance in schizophrenia patients. J Clin Psychiatry 2003;64(16):10-13.

16. Morken G, Widen JH, Grawe RW. Non-adherence to antipsychotic medication, relapse and rehospitalisation in recent-onset schizophrenia. BMC Psychiatry 2008;8:32-34.

17. Borras L, Mohr S, Brandt PY, Gilliéron C et al. Religious beliefs in schizophrenia: Their relevance for adherence to treatment. Schizophr Bull 2007;33(5):1238-1246.

18. Eaddy M, Grogg A, Locklear J. Assessment of compliance with antipsychotic treatment and resource utilization in a Medicaid population. Clin Ther 2005;27:263-272.

19. Robinson D, Woerner MG, Alvir JM et al. Predictors of relapse following response from a first episode of schizophrenia or schizoaffective disorder. Arch Gen Psychiatry 1999;56:241-247.

20. Weiden PJ, Olfson M. Cost of relapse in schizophrenia. Schizophr Bull 1995;21:419-429.

21. Czobor P, Van Dorn RA, Citrome L, Kahn RS et al. Treatment adherence in schizophrenia: a patient-level meta-analysis of combined CATIE and EUFEST studies. Eur Neuropsychopharmacol 2015; 25(8):11581166.

22. Opolka, JL, Rascati KL, Brown CM, Gibson PJ. Role of ethnicity in predicting antipsychotic medication adherence. Ann Pharmacother 2003a;37(5):625-630.

23. Opolka JL, Rascati KL, Brown, CM, Barner JC et al. Ethnic differences in use of antipsychotic medication among Texas medicaid clients with schizophrenia. J Clin Psychiatry 2003b;64(6):635-639.

24. Diaz E, Woods SW, Rosenheck R. Effects of Ethnicity on Psychotropic Medications Adherence. Community Ment Health J 2005;41:521-537.

25. Gilmer TP, Dolder CR, Lacro JP et al. Adherence to treatment with antipsychotic medication and health care costs among Medicaid beneficiaries with schizophrenia. Am J Psychiatry 2004;161:692-699.

26. Valencia M, Rascon ML, Juarez F, Murow E. A psychosocial skills training approach in Mexican out-patients with schizophrenia. Psychol Med 2007;37(10):1393-1402.

27. Valencia $M$, Rascon ML, Juarez F, Escamilla $R$ et al. Application in
Mexico of psychosocial rehabilitation with schizophrenia patients. Psychiatry 2010;73(3):248-263.

28. Valencia M, Juarez F, Ortega H. Integrated treatment to achieve functional recovery for first-episode psychosis. Schizophr Res Treatment 2012; doi:10.1155/2012/962371

29. Valencia M, Fresan A, Juárez F, Escamilla $R$ et al. The beneficial effects of combining pharmacological and psychosocial treatment on remission and functional outcome in outpatients with schizophrenia. J Psychiatric Research 2013;47(12):1886-1892.

30. Valencia M, Moriana JA, Kopelowicz A, Lopez SR et al. Social-Skills training for Spanish-speaking persons with schizophrenia: Experiences from Latin America, Spain, and the United States. Am J Psychiatr Rehabil 2015;18(3):209-246.

31. Hogan TP, Awad AG, Eastwood R. A self-report scale predictive of drug compliance in schizophrenics: reliability and discriminative validity. J Psychol Med 1983;13:177-183.

32. Barnes TR. A rating scale for drug-induced akathisia. Br J Psychiatry 1989;154:672-676.

33. Kay SR, Fiszbein A, Opler L. The positive and negative syndrome scale (PANSS) for schizophrenia. Schizophr Bull 1987;13:261-276.

34. Köster G. Los Aymaras: Características demográficas de un grupo étnico indígena antiguo en los Andes centrales. En: Van den Berg $\mathrm{H}$, Schiffers N (eds.). La cosmovisión Aymara La Paz, Bolivia: UCB/ Hisbol; 1992; pp. 81-111.

35. Van Kessel J. La cosmovisión Aymara. En: Hidalgo J, Schiappacasse F, Niemeyer F, Aldunate C, Mege P. (eds.). Etnografía: Sociedades indígenas contemporáneas y su ideología. Santiago, Chile: Editorial Andrés Bello; 1996; pp:169-187.

36. Gundermann H. Las organizaciones étnicas y el discurso de la identidad en el norte de Chile, 1980-2000. Estudios Atacameños 2000;19:75-91.

37. Zapata C. Memoria e historia: El proyecto de una identidad colectiva entre los aymaras de Chile. Chungara 2007;39:171-183.

38. De Munter K. Tejiendo reciprocidades: John Murra y el contextualizar entre los aymara contemporáneos en Chungara. Chungara 2010;4:243251.

39. Juntuma de Chapiquiña. Medicina indígena y alimentación tradicional del pueblo de Chapiquiña. Trabajo fundando por el Programa de Salud y Pueblos Indígenas (PEPSI); 2002.

40. Laks, Jordana. Reflexiones sobre la tristeza y la preocupación en las personas Aymara de Putre, Chile. Independent Study Project (ISP) Collection. Paper 1054; 2011. http://digitalcollections.sit.edu/isp_collection/1054. Accessed March 19, 2013.

41. World Health Organization. ICD-10 Classifications of Mental and Behavioural Disorder: Clinical Descriptions and Diagnostic Guidelines. Ginebra, Suiza: World Health Organization; 1992.

42. Nielsen RE, Lindström E, Nielsen J, Levander S. DAI-10 is as good as DAI-30 in schizophrenia. Eur Neuropsychopharmacol 2012;22(10):747750.

43. Saleem F, Hassali MA, Shafie AA, Awad AG et al. Association between knowledge and drug adherence in patients with hypertension in Quetta, Pakistan. Trop J Pharm Res 2011;10:125-132.

44. Ramírez Barreto F, Robles García R, Salazar Alvarado V, Páez Agraz F. Evaluación de actitudes al medicamento en pacientes con esquizofrenia: propiedades psicométricas de la versión en español del DAI. Actas Esp Psiquiatr 2004;32(3):138-142.

45. Peralta V, Cuesta MJ. Validación de la Escala de los Síndromes Positivo y Negativo (PANSS) en una muestra de esquizofrénicos españoles. Actas Luso Esp Neurol Psiquiatr Cienc Afines 1994;22(4):171-177.

46. Fresán A, De la Fuente-Sandoval C, Loyzaga C, García-Anaya, M et al. A forced five-dimensional factor analysis and concurrent validity of the Positive and Negative Syndrome Scale in Mexican schizophrenic patients. Schizophr Res 2005;72:123-129.

47. Markkula N, Alvarado R, Minoletti A. Adherence to guidelines and treatment compliance in the Chilean national program for first-episode schizophrenia. Psychiatr Serv 2011;62(12):1463-1469. 
48. Caqueo-Urízar A, Breslau J, Gilman S. Beliefs about the causes of schizophrenia among Aymara and non-Aymara patients and their primary caregivers in the Central-Southern Andes. Int J Soc Psychiatry 2015;61(1):82-91.

49. Gundermann H. Acerca de cómo los aymaras aprendieron el castellano (terminando por olvidar el aymara). Estudios Atacameños 1997;12:89104.

50. Gavilán V, Vigueras P, Carrasco A, Cabezas R et al. Pautas de crianza Aymara. Significaciones, actitudes y prácticas de familias Aymara en relación a la crianza y cuidado infantil de los niños y niñas desde la gestación hasta los diez. Chile: Centro de Investigaciones para el Desarrollo del Hombre en el Desierto, Universidad Arturo Prat, Chile; 2006.

51. Tsai JK, Lin WK, Lung FW. Social interaction and drug attitude effectiveness in patients with schizophrenia. Psychiatr Q 2011;82(4):343-51.

52. Yang J, Ko Y-H, Paik J-W et al. Symptom severity and attitudes toward medication: Impacts on adherence in outpatients with schizophrenia. Schizophrenia Res 2012;134(2-3):226-231.

53. Gaebel W, Riesbeck M, von WM, Burns $T$ et al. Drug attitude as Predictor for effectiveness in first-episode schizophrenia: results of an open randomized trial (EUFEST). Eur Neuropsychopharmacol 2010;20:310-316.

54. Mohamed S, Rosenheck R, McEvoy J et al. Crosssectional and longitudinal relationships between insight and attitudes toward medication and clinical outcomes in chronic schizophrenia. Schizophr Bull 2009;35(2):336-346.

55. Nakonezny PA, Byerly MJ, Rush AJ. Electronic monitoring of antipsychotic medication adherence in outpatients with schizophrenia or schizoaffective disorder: an empirical evaluation of its reliability and predictive validity. Psychiatry Res 2008;157:259-263.

56. Sellwood W, Tarrier N, Quinn J et al. The family and compliance in schizophrenia: the influence of clinical variables, relatives' knowledge and expressed emotion. Psychol Med 2003;33:91-96.

57. Freudenreich O, Cather C, Evins A, Henderson D et al. Attitudes of schizophrenia outpatients toward psychiatric medications: relationship to clinical variables and insight. J Clin Psychiatry 2004;65:1372-6.

58. Nosé M, Barbui C, Tansella M. How often do patients with psychosis fail to adhere to treatment programmes? A systematic review. Psychol Med 2003;33:1149-1160.

59. Sajatovic M, Valenstein M, Blow F, Ganoczy D et al. Treatment adherence with lithium and anticonvulsant medications among patients with bipolar disorder. Psychiatr Serv 2007;58(6):855-863.

60. Perkins DO, Johnson JL, Hamer RM, Zipursky RB et al. Predictors of antipsychotic medication adherence in patients recovering from a first psychotic episode. Schizophr Res 2006;83(1):53-63.

61. Perkins DO, Gu H, Weiden PJ, McEvoy JP et al. Predictors of treatment discontinuation and medication nonadherence in patients recovering from a first episode of schizophrenia, schizophreniform disorder, or schizoaffective disorder: a randomized, double-blind, flexible-dose, multicenter study. J Clin Psychiatry 2008;69(1):106-113.

62. Vassileva IV, Milanova VK. Attitudes toward antipsychotic medication, insight and psychopathology in outpatients with schizophrenia. Folia Med 2012;54(4):62-68. 